\title{
Faster male displays and less complex choice are more attractive to female fiddler crabs as they reduce search costs
}

\author{
Fábio Henrique Carretero Sanches ${ }^{\text {a, * }}$, Tânia Marcia Costa ${ }^{\text {b }}$, Rodrigo Egydio Barreto a , \\ Patricia R. Y. Backwell ${ }^{\mathrm{c}}$ \\ ${ }^{a}$ Department of Physiology, Botucatu Biosciences Institute, São Paulo State University (UNESP), Botucatu, SP, Brazil \\ ${ }^{\mathrm{b}}$ Biosciences Institute, Coastal Campus, São Paulo State University (UNESP), São Vicente, SP, Brazil \\ ${ }^{\mathrm{c}}$ Research School of Biology, The Australian National University, Canberra, ACT, Australia
}

\section{A R T I C L E I N F O}

\section{Article history:}

Received 13 July 2016

Initial acceptance 8 September 2016

Final acceptance 26 October 2016

Available online 11 January 2017

MS. number: A16-00623R

\section{Keywords:}

accuracy

mate choice

robotic crab

sexual selection

waving display

\begin{abstract}
Females that quickly and accurately locate and assess males can reduce their risks of predation, dehydration and heat stress while mate searching. Here we measured the accuracy and time it took female fiddler crabs, Uca mjoebergi, to approach robotic claws that simulated males' courtship signals. We ran six experiments: three one-choice experiments varying in waving display rate (fast, medium and slow) and three three-choice experiments with increased number of displays (all with fast wave rate) and complexity (each one at the three different rates; and the three different rates presented at different distances, with the fast wave rate further from the female and the slow wave rate closer to the female). Females approached all waving robots with an accuracy of 9-18 ${ }^{\circ}$. They approached faster-waving claws more quickly even when they were presented in sets of three claws, but it took females longer to approach a claw in the more complex situation, with claws waving at different rates and distances. Females may approach waving claws more rapidly simply because they present a more continuous and less ambiguous stimulus. The results suggest that high signalling rates may attract females because they reduce female search costs, and they may or may not additionally signal male quality.
\end{abstract}

(c) 2016 Published by Elsevier Ltd on behalf of The Association for the Study of Animal Behaviour.
Mate choice is expensive for females. Any increase in searching time will further increase her costs (energy, predation risk, dehydration, overheating). If a male's signal is difficult to localize, a female would take a more circuitous path to the signaller and spend more time exposed to risks. It is not surprising, therefore, that many animals are able to localize signals with great precision. Females usually follow a zigzag path towards displaying males, and the average error of each movement from the target axis is used to estimate the precision of approach. Most female anurans have an approach error angle of $16-23^{\circ}$, but one species has an accuracy of $1^{\circ}$ (Rheinlaender, Gerhardt, Yager, \& Capranica, 1979; Shen et al., 2008; Ursprung, Ringler, \& Hödl, 2009); crickets have an accuracy of 10-14 (Schöneich \& Hedwig, 2010); a fly was shown to have an accuracy of $1-2^{\circ}$ (Mason, Oshinsky, \& Hoy, 2001).

By making his signal stand out, a male can make himself more detectable and more locatable, and this can attract more females (Mowles \& Ord, 2012; Ryan \& Cummings, 2005; Wilson \& Mennill,

\footnotetext{
* Correspondence: F. H. C. Sanches, Department of Physiology, Botucatu Biosciences Institute, São Paulo State University (UNESP), Botucatu, SP, Brazil.

E-mail address: fabiohcsanches@yahoo.com.br (F. H. C. Sanches).
}

2011). In many species, the same male traits that increase the conspicuousness or locatability of a signaller may also signal his quality or act as a handicap (Mowles \& Ord, 2012; Ryan \& Cummings, 2005). High signalling rate is one example: it is expensive for males to signal at a high rate (time, energy and predation risk), so display rate is often considered to signal male quality or act as a handicap (Mowles \& Ord, 2012; Ryan \& Cummings, 2005). In field crickets, males that signal more rapidly accumulate greater energetic costs, and it was suggested that females select mates based on their ability to bear these costs (Mowles, 2014). In chickadees, a slow display rate prevented females from locating the stimulus, and a high display rate caused females to approach the speakers more quickly (Wilson \& Mennill, 2011). By increasing the display rate when a female is detected, a male may make himself more visible and more locatable. In a fiddler crab, for example, males increase their wave rate when they detect wandering females (or when they detect the increased wave rate of other males that have seen a female); this increases their conspicuousness and consequently elevates their likelihood of being approached by the female (Milner, Jennions, \& Backwell, 2010). If the signal functions entirely to facilitate male localization, it would still be energetically expensive and males would still 
succumb to the energetic costs, but females would approach the more rapidly waving males simply because they are more locatable. There may or may not be additional benefits (e.g. females that mate with more easily detected males may produce sons that are also more easily detected), meaning that the locatability of a signal and its possible role in mate assessment may be closely linked.

Enhanced locatability of complex over simple calls has been suggested as a potential reason why females strongly prefer complex calls in the túngara frog, Physalaemus pustulosis (Bonachea \& Ryan, 2011). It was shown that females chose more quickly when presented with complex calls than when listening to simple calls, but the accuracy of approach was no different between simple and complex calls (Bonachea \& Ryan, 2011). Female tree frogs were also found to approach complex three-component and simpler onecomponent calls with equal accuracy (Rheinlaender et al., 1979). In the leaf-folding frog, Afrixalus delicatus, neither the approach accuracy nor the time to reach the signal were affected by call complexity or number of males present (Backwell \& Passmore, 1991).

All the above studies were on acoustically communicating species. This probably reflects the ease with which sound signals can be manipulated and phonotaxis experiments conducted. Visual signals are more difficult since they often require the use of robotic models or video presentations of courtship displays. We know of no study that has examined the accuracy of mate attraction to visual/ movement-based signals that differ in signalling rate, signal complexity or the number of signallers present. Here we use robotics to examine the accuracy and speed of female approaches to signals in the movement-based courtship of a fiddler crab. We test the effect of display rate and choice complexity on the accuracy and speed of female approach. We specifically ask whether the accuracy or duration of female approaches are affected by (1) wave rate, (2) the number of waving claws or (3) the complexity of the choice context (variation in signals and distances).

\section{METHODS}

We studied a population of the fiddler crab Uca mjoebergi from September to December 2015 at East Point Reserve, Darwin, Australia $\left(12^{\circ} 24^{\prime} 31.89^{\prime \prime} \mathrm{S}, 130^{\circ} 49^{\prime} 49.12^{\prime \prime} \mathrm{E}\right)$. Uca mjoebergi is a small fiddler crab (mean \pm SD carapace width $=10.16 \pm 1.43 \mathrm{~mm}$; $N=200$ ) that occurs on the northern coast of Australia. Both males and females defend territories within a large, mixed-sex population. A territory consists of a small area of sediment surface with a central burrow. Males court females from the surface around their burrow by waving their enlarged claw. When a female is ready to mate, she will leave her territory and move through the population of waving males. Males form small clusters (2-6) around the female and, as she moves, males join in or drop out of the cluster. The female visits one of the males in the cluster by walking directly towards him and briefly entering his burrow. She then either leaves the male to continue searching, or she accepts the male and remains underground in his burrow. The chosen male enters the burrow and plugs its entrance with sand; mating occurs within $1 \mathrm{~h}$. The male remains underground with the female, guarding her until she extrudes her eggs onto her pleopods $1-5$ days later. The female is then unable to remate, and the male leaves, resealing her in the burrow.

Female preferences were tested using custom-built robotic crabs consisting of a twin-cam motor that moved a small metal arm in a motion exactly mimicking the courtship wave of the species. The motor is remotely controlled to regulate the exact timing of each wave using custom-designed software (for further details of the robotic crabs, see Booksmythe, Detto, \& Backwell, 2008; Holman, Kahn, \& Backwell, 2014; Reaney, Sims, Sims, Jennions, \&
Backwell, 2008). The motor was buried under the testing arena with only the metal arm protruding through the arena floor. The arm had a plaster replica of $U$. mjoebergi claw attached to it. For all trials, we used replicas of the same claw, each measuring $24 \mathrm{~mm}$ and painted a yellow that matched the natural claw colour of this species (for details of the claw and paint colour, see Detto, Backwell, Hemmi, \& Zeil, 2006). The choice arena was a cleared area of mudflat that was levelled to provide a uniform surface. We placed a video camera (Sony DCR-SR65E) directly above the centre of the arena so that we could film an area of $45 \times 45 \mathrm{~cm}$ of the choice arena.

Mate-searching females were captured as they wandered through the population of courting males. We housed them individually in shaded cups containing $0.2 \mathrm{~cm}$ deep sea water until we used them in the choice trials. For each trial, the female was placed at the release point on one end of the test arena, in a small translucent cup that was remotely lifted once the female had seen three waves of the robotic crabs (for more details, see Booksmythe et al., 2008; Reaney, 2009). A positive response was scored when the female touched (or approached to within $5 \mathrm{~cm}$ ) a robotic crab arm. Trials were discarded if the female darted, ran to the edge of the area, or remained stationary for $>3 \mathrm{~min}$. Each female was retested up to a maximum of three times (each in a different experiment and in a random order), but females were never tested in the same trial more than once. Females were released after they were tested so they could continue mate searching. Females naturally visit numerous males so it is not unreasonable to test them in multiple trials.

We tested 20 females in each of six experiments, and filmed each trial. We analysed the videos using ImageJ (National Institutes of Health, Bethesda, MD, U.S.A.). Trial durations were measured as the time from female release (lifting the translucent cup) until the female reached the robotic claw. We calculated the error angle of each trial by dividing the area between the release point and the robotic crabs into four sections, each $5 \mathrm{~cm}$ long. Then, we marked the female's position as she crossed each line and calculated the angle as the difference between (1) the line joining the female with the robotic claw (target axis) and (2) the line joining the female at position $n$ with her position at $n+1$ (the jump axis between successive lines; Fig. 1). This resulted in three error angles (Fig. 1). To

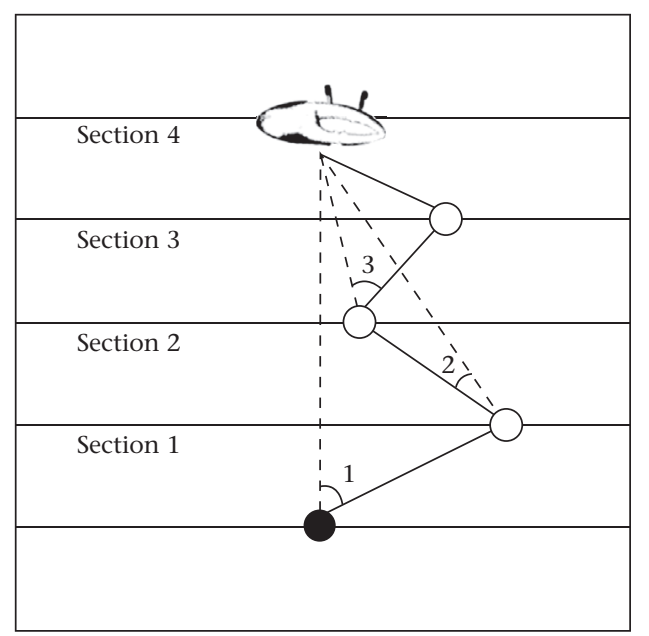

Figure 1. Experimental design with robotic crab (male claw) and female positions (the black circle is the release point and the white circles are the positions when she crossed the lines that divided the arena into four sections of $5 \mathrm{~cm}$ ). Error angles (1, 2 and 3 ) were calculated from the angle between (1) a straight line from the female starting position to the robotic crab (dotted connecting lines) and (2) the line between the female starting position and the female position at the next line (solid connecting lines). 
make the readings comparable to published work (Murphey \& Zaretsky, 1972; Rheinlaender et al., 1979), we summed the three error angles and divided the total by four (since the final error angle is necessarily zero). This gave the 'accuracy' score for each female.

\section{Wave Rate Experiments}

We ran three one-choice experiments to determine the effect of wave rate on the duration and accuracy of female approaches. In all three, the female was released $20 \mathrm{~cm}$ away from the robotic crab. For the fast wave rate experiment $(\mathrm{F})$, the claw waved at 16.8 waves/min. For the medium wave rate experiment $(M)$, the claw waved at 8.4 waves/min, and for the slow wave rate experiment $(\mathrm{S})$, the claw waved at 4.2 waves $/ \mathrm{min}$. We tested 20 females in each experiment.

\section{Choice Complexity Experiments}

We ran three three-choice experiments with increasing complexity to determine their effect on the duration and accuracy of female approaches. In the least complex experiment (FFF), we presented the female with three robotic claws in an arc $20 \mathrm{~cm}$ in front of and directly facing her. All three waved at the fast rate (16.8 waves/min). In the mid-level complexity experiment (FMS), we presented the female with three robotic claws in an arc $20 \mathrm{~cm}$ in front of and directly facing her. One claw waved at the fast rate ( 16.8 waves $/ \mathrm{min}$ ), one at the medium rate $(8.4$ waves $/ \mathrm{min}$ ) and one at the slow rate ( 4.2 waves/min). The most complex trial (FMSdd) was the same as the previous trial but with the stimuli presented at different distances. The fast-waving claw was placed $22 \mathrm{~cm}$ away from the female release point; the medium-waving claw was placed at $20 \mathrm{~cm}$; and the slow-waving claw was placed at $18 \mathrm{~cm}$. We tested 20 females in each experiment.

In the three-choice trials, we measured the duration and accuracy of the female approach to her chosen robotic male. In most cases, the female approached the fastest waving male, but in $7 / 60$ trials the female approached either the medium or slow waving robot.

\section{Statistical Analysis}

We analysed the first three (one-choice) trials using a multivariate general linear model (MGLM) with trial duration and accuracy as the dependent variables, female carapace width as a covariate and experiment type (F, M or $\mathrm{S}$ ) as a fixed factor. The standardized residuals were normally distributed and the scatterplot of predicted values against residuals had a shotgun pattern. To interpret the multivariate test results, we examined its univariate components (duration and error angle).

We examined the effect of 'number of waving claws' by comparing the trial duration and accuracy between the fast-waverate one-choice trial and the fast-wave-rate three-choice trial ( $F$ versus FFF). We used an MGLM as above.

We analysed the last three experiments (three-choice trials) in the same way. Since the females did not always select the fast waving male, we calculated the difference in duration and accuracy in the three-choice trial with the associated mean duration and accuracy for the one-choice trials. If the female approached the slow waving robot in the three-choice trial, we subtracted the mean duration and accuracy score of the one-choice 'slow' trials from her duration and accuracy scores in the three-choice trial. If the female approached the fast waving robot in the three-choice trial, we subtracted the mean duration and accuracy score of the one-choice 'fast' trials from her duration and accuracy scores in the threechoice trial. If the female approached the medium waving robot in the three-choice trial, we subtracted the mean duration and accuracy score of the one-choice 'medium' trials from her duration and accuracy scores in the three-choice trial. This gave us a measure of the change in duration and accuracy between the simple onechoice trial and the more complex three-choice trial. In the trials where claws were presented at different distances (FMSdd), all females selected the fast-waving claw and it was presented at $22 \mathrm{~cm}$ away from the female $(2 \mathrm{~cm}$ further than in all other trials).

We analysed 'difference in duration and accuracy' for the three complex trials using a multivariate general linear model (MGLM) with duration difference and accuracy difference as the dependent variables, female carapace width as a covariate and experiment type (FFF, FMS or FMSdd) as a fixed factor. The standardized residuals were normally distributed and the scatterplot of predicted values against residuals had a shotgun pattern. To interpret the multivariate test results, we examined its univariate components (duration and accuracy).

Statistical analyses were conducted in SPSS version 23.0 (SPSS Inc., Armonk, NY, U.S.A.) and the alpha level was set at $P=0.05$.

\section{Ethical Note}

No ethics permit was required for this study. We limited the handling and the amount of time each crab was used as much as possible. No crab was injured during the research, and they all continued their regular activities after release. The work was conducted under a research permit from the Darwin City Council (permit no. 2322876).

\section{RESULTS}

\section{Effect of Wave Rate on Accuracy and Duration}

An MGLM showed that female size did not affect the accuracy or duration of approach (Hotelling's trace: $F_{2,55}=0.31, P=0.73$; see Table 1 for female sizes). Wave rate did, however, have an effect (Hotelling's trace: $F_{4,108}=7.20, P<0.001$; see Table 1 for descriptive statistics). The effect was driven by differences in the approach durations (univariate effects: duration: $F_{2,56}=14.14, P<0.001$; accuracy: $\left.F_{2,56}=0.56, P=0.58\right)$. Females took longer to approach when the wave rate was slower, but their approach was equally accurate at all wave rates (Fig. 2).

\section{Effect of Number of Waving Claws on Accuracy and Duration}

An MGLM showed that female size did not affect the accuracy or duration of approach (Hotelling's trace: $F_{2,36}=0.85, P=0.43$ ). The number of robotic waving claws (one or three robots) did not affect the approach accuracy or trial duration (Hotelling's trace:

Table 1

Descriptive statistics (means $\pm \mathrm{SD}$ ) for female size (carapace width), trial duration and accuracy for the six experiments

\begin{tabular}{llll}
\hline Experiment & Female size $(\mathrm{mm})$ & Duration $(\mathrm{s})$ & Accuracy (degrees) \\
\hline F & $8.82 \pm 0.83$ & $19.25 \pm 13.44$ & $9.94 \pm 7.14$ \\
M & $8.97 \pm 0.95$ & $64.25 \pm 42.20$ & $12.16 \pm 9.22$ \\
S & $9.15 \pm 0.94$ & $109.45 \pm 82.43$ & $9.73 \pm 7.51$ \\
FFF & $8.95 \pm 0.90$ & $23.50 \pm 16.22$ & $11.28 \pm 5.81$ \\
FMS & $8.96 \pm 0.91$ & $32.40 \pm 28.90$ & $17.80 \pm 11.88$ \\
FMSdd & $8.95 \pm 0.86$ & $44.05 \pm 32.46$ & $15.35 \pm 8.83$ \\
\hline
\end{tabular}

$\mathrm{F}=$ one-choice test with fast wave rate; $\mathrm{M}=$ one-choice test with medium wave rate; $\mathrm{S}=$ one-choice test with slow wave rate; $\mathrm{FFF}=$ three-choice test all with fast wave rate; FMS = three-choice test with one fast, one medium and one slow wave rate; FMSdd = three-choice test with one fast, one medium and one slow wave rate, presented at different distances (fast $=22 \mathrm{~cm}$; medium $=20 \mathrm{~cm}$; slow $=18 \mathrm{~cm}$ ). 

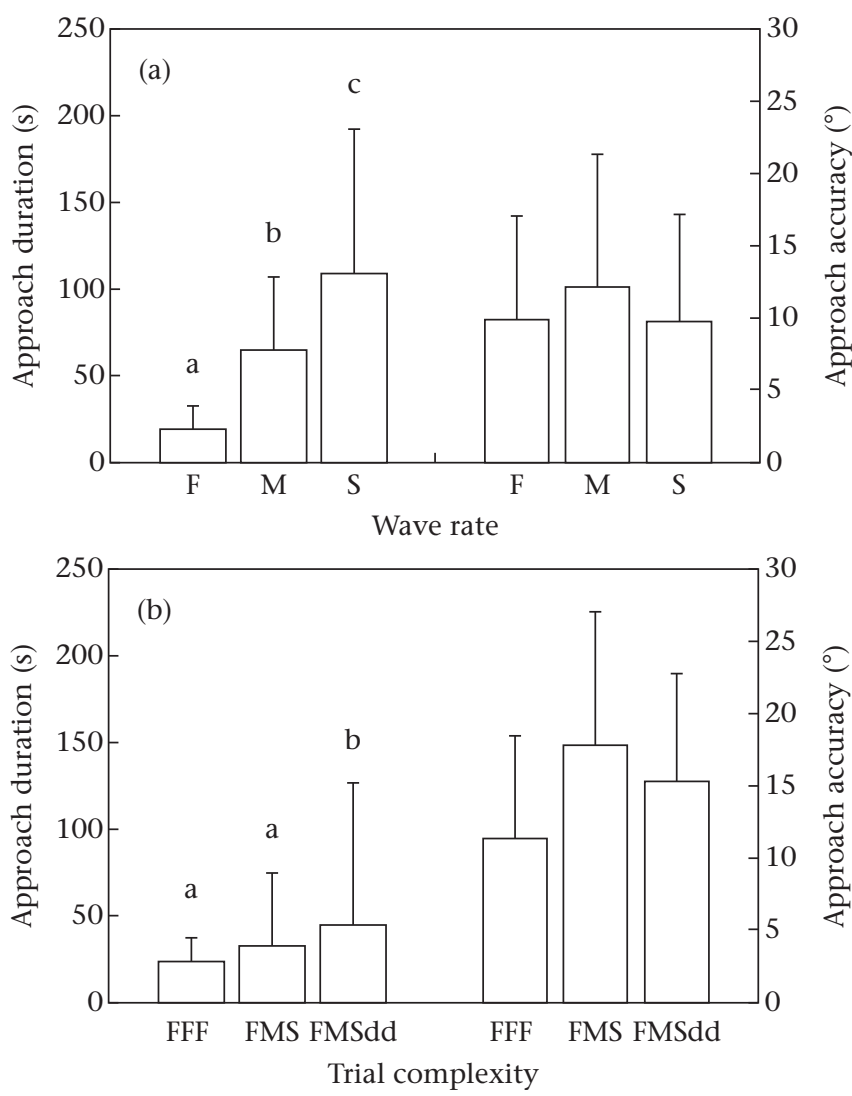

Figure 2. Approach duration and accuracy for (a) one-choice trials that differed in wave rate and (b) three-choice trials that differed in complexity for the six experiments: $\mathrm{F}=$ one-choice test with fast wave rate; $\mathrm{M}=$ one-choice test with medium wave rate; $S=$ one-choice test with slow wave rate; $F F=$ three-choice test all with fast wave rate; FMS = three-choice test with one fast, one medium and one slow wave rate; FMSdd = three-choice test with one fast, one medium and one slow wave rate, presented at different distances (fast $=22 \mathrm{~cm}$; medium $=20 \mathrm{~cm}$; slow $=18 \mathrm{~cm}$ ). Different letters above bars denote statistical differences $(P<0.05)$.

$F_{2,36}=0.71, P=0.50 ;$ univariate effects: duration: $F_{1,37}=0.92$, $P=0.35$; accuracy: $F_{1,37}=0.32, P=0.57$ ). The female approach was equally accurate and took the same amount of time when there was a single waving claw and when there were three waving claws.

\section{Effect of Choice Complexity on Accuracy and Duration}

An MGLM showed that female size did not affect the accuracy or duration of approach (Hotelling's trace: $F_{2,55}=0.86, P=0.43$; see Table 1 for female sizes). The complexity of the choice arena did, however, have an effect (Hotelling's trace: $F_{4,108}=3.04, P=0.02$; see Table 1 for descriptive statistics). The effect was driven by differences in the approach durations rather than approach accuracy (univariate effects: duration: $F_{2,56}=3.91, P=0.03$; accuracy: $\left.F_{2,56}=2.34, P=0.11\right)$. Females took longer to approach when the choices were more complex, but their approach was equally accurate irrespective of the wave rate (Fig. 2).

\section{DISCUSSION}

\section{Accuracy of Localization}

Female $U$. mjoebergi approached the waving claw of the robotic male crabs with an accuracy of $9-18^{\circ}$. To our knowledge, this is the first documentation of approach accuracy to a movement-based visual signal, so it is interesting that the level of error was equivalent to most frogs $\left( \pm 20^{\circ}\right.$; Rheinlaender et al., 1979; Shen et al., 2008) and crickets $\left( \pm 12^{\circ}\right.$; Schöneich \& Hedwig, 2010). However, the approach accuracy measured here may be considerably less than the accuracy with which females approach males in the field: males of many species (including the study species) move towards a female and 'lead' her back to their burrow by waving at an elevated rate and walking backward to allow her to follow (Crane, 1975; How, Hemmi, Zeil, \& Peters, 2008). This 'leading' behaviour may increase female approach accuracy under natural conditions.

The accuracy of approach was not affected by the wave rate: females approached a slow-waving claw as accurately as a fastwaving claw. The complexity of the choice scenario also did not affect approach accuracy: females approached a single stimulus as accurately as they approached one presented alongside two others that waved at different rates and were at different distances from the female. This result has also been found in other species: the repetition rate of a dendrobatid frog did not affect the accuracy of approach (Ursprung et al., 2009); neither did the call complexity affect accuracy in three frog species (Backwell \& Passmore, 1991; Bonachea \& Ryan, 2011; Rheinlaender et al., 1979). Although simultaneous calling by neighbouring frogs was predicted to reduce their locatability (Awbrey, 1978), female approach accuracy in the painted reed frog, Hyperolius marmoratus, was unaffected by call overlap (Passmore \& Telford, 1981). The accuracy with which a female approached a calling male in a pond, under natural conditions, was no different to the accuracy in controlled, singlestimulus laboratory trials (leaf-folding frog: Backwell \& Passmore, 1991; dendrobatid frog: Gerhardt, 1980). Even in a non-matesearching context, the accuracy of approach by a parasitoid fly to its hosts' (cricket) calls was only slightly less accurate when the repetition rate was halved (Muller \& Robert, 2002).

It does not appear that repetition rate or the complexity of the signal or choice environment influences the accuracy with which a female can locate a stimulus. This is surprising and it suggests that the accuracy of localization is constrained by the female's sensory system (see Bonachea \& Ryan, 2011; Ursprung et al., 2009).

\section{Time to Locate}

In contrast, the time it took for female $U$. mjoebergi to approach the waving claw was significantly affected by wave rate: it took females \pm 110 s to approach a slow-waving claw; \pm 64 s to approach a claw waved at a medium rate; and only \pm 19 s to approach a fastwaving claw. The number of stimuli did not affect the approach time: females took just as long to approach a fast-waving claw when it was presented alone or with two additional fast-waving claws. However, when the context of choice was more complex, females took longer to approach the claw: it took $24 \mathrm{~s}$ for the female to approach when three identical, fast-waving stimuli were presented; it took an additional $10 \mathrm{~s}$ when the stimuli had three different wave rates; and a further $12 \mathrm{~s}$ more when the stimuli were at different distances from the female. Although the approach distance in the FMSdd trials was $2 \mathrm{~cm}$ further than in other trials, this $10 \%$ increase in distance cannot explain the $36 \%$ increase in the time taken.

It is not surprising that it took females longer to locate a signal with a low repetition rate since there are fewer waves per unit time to guide her approach and allow her to make corrections to her approach path rapidly. In a dendrobatid frog, females also took less time to approach a rapidly repeated call than a slower call, but this was because they only jumped towards the sound source during signal production and not during the intercall intervals (Ursprung et al., 2009). An increase in signal repetition rate does not always decrease the time it takes to reach a signal source: repetition rate had no effect on approach time in the leaf-folding frog (Backwell \& 
Passmore, 1991) or in the approach of a parasitoid fly to the calls of its host (Muller \& Robert, 2002).

The effect of choice complexity on the approach time is similarly inconsistent in other species. Female túngara frogs approached complex calls more quickly than simple calls (Bonachea \& Ryan, 2011); but in the leaf-folding frog, females were able to locate a naturally calling male in a pond just as quickly as a single stimulus presented under highly controlled experimental conditions (Backwell \& Passmore, 1991).

\section{Conclusions}

Finding a mate is an expensive process, and females need to quickly and accurately detect a male, locate him and then (possibly) assess his suitability as a mate. Any increase in the time a female must spend to achieve this will increase her exposure to risks (predation, dehydration, overheating, etc.). We suggest that, while a female's ability to increase the accuracy of localization would be constrained by her sensory system, she is able to decrease her approach time by selecting signals with high repetition rates and less complex choice environments.

Males could increase their chances of mating by signalling as fast as possible. When females are unpredictable in space and time, vigorous and constant display may be costly (Ryan \& Cummings, 2005). High signalling rates may attract females because they reduce the female search costs; and they may or may not additionally signal male quality. In $U$. mjoebergi, males with fast wave rates are preferentially approached by mate-searching females (Callander, Jennions, \& Backwell, 2012), possibly because wave rate signals male quality, but probably also (or even entirely) because faster wave rates can be located more quickly.

\section{Acknowledgments}

We thank the North Australian Research Unit for facilities and Nina Svedin and Daniela M. Perez for the valuable help in the field work. This research received financial support from an Australian Research Council Discovery Grant (ARC DP120101427). F. H. C. Sanches received financial support through a scholarship from PDSE - CAPES (Process: 99999.003008/2015-00).

\section{References}

Awbrey, F. T. (1978). Social interaction among chorusing Pacific tree frogs, Hyla regilla. Copeia, 1978, 208-214.
Backwell, P. R. Y., \& Passmore, N. I. (1991). Sonic complexity and mate localization in the leaf-folding frog, Afrixalus delicatus. Herpetologica, 47, 226-229.

Bonachea, L. A., \& Ryan, M. J. (2011). Localization error and search costs during mate choice in túngara frogs, Physalaemus pustulosis. Ethology, 117, 56-62.

Booksmythe, I., Detto, T., \& Backwell, P. R. Y. (2008). Female fiddler crabs settle for less: Cost-benefit trade-offs in mate choice. Animal Behaviour 76, 1775-1781.

Callander, C., Jennions, M. D., \& Backwell, P. R. Y. (2012). The effect of claw size and wave rate on female choice in a fiddler crab. Journal of Ethology, 30, 151-155.

Crane, J. H. (1975). Fiddler crabs of the word. Ocypodidae: Genus Uca. Princeton, NJ: Princeton University Press.

Detto, T., Backwell, P. R. Y., Hemmi, J., \& Zeil, J. (2006). Visually mediated species and neighbour recognition in fiddler crabs (Uca mjoebergi and Uca capricornis). Proceedings of the Royal Society B: Biological Sciences, 273, 1661-1666.

Gerhardt, H. C. (1980). Accuracy of sound localization in a miniature dendrobatid frog. Naturwissenschaften, 67, 362-363.

Holman, L., Kahn, A., \& Backwell, P. R. Y. (2014). Fiddlers on the roof: Elevation muddles mate choice in a fiddler crab. Behavioral Ecology, 25, 271-275.

How, M. J., Hemmi, J. M., Zeil, J., \& Peters, R. (2008). Claw waving display changes with receiver distance in fiddler crabs, Uca perplexa. Animal Behaviour, 75, 1015-1022.

Mason, A. C., Oshinsky, M. L., \& Hoy, R. (2001). Hyperacute directional hearing in a microscale auditory system. Nature, 410, 686-688.

Milner, R. N. C., Jennions, M. D., \& Backwell, P. R. Y. (2010). Eavesdropping in crabs: An agency for lady detection. Biology Letters, 6, 755-757.

Mowles, S. L. (2014). The physiological cost of courtship: Field cricket song results in anaerobic metabolism. Animal Behaviour, 89, 39-43.

Mowles, S. L., \& Ord, T. J. (2012). Repetitive signals and mate choice: Insights from contest theory. Animal Behaviour, 84, 295-304.

Muller, P., \& Robert, D. (2002). Death comes suddenly to the unprepared: Signing crickets, call fragmentation, and parasitoid flies. Behavioral Ecology, 13, 598-606.

Murphey, R. K., \& Zaretsky, M. D. (1972). Orientation to calling song by female crickets Scapsipedus marginatus (Gryllidae). Journal of Experimental Biology, 56, $335-352$.

Passmore, N. I., \& Telford, S. R. (1981). The effect of chorus organization on mate localization in the painted reed frog (Hyperolius marmoratus). Behavioral Ecology and Sociobiology, 9, 292-293.

Reaney, L. T. (2009). Female preferences for male phenotypic traits: Do females use absolute or comparative evaluation? Animal Behaviour, 77, 139-143.

Reaney, L., Sims, R. A., Sims, S. W. M., Jennions, M. D., \& Backwell, P. R. Y. (2008). Experiments with robots explain synchronized courtship in a fiddler crab. Current Biology, 18(2), 62-63.

Rheinlaender, J., Gerhardt, H. C., Yager, D. D., \& Capranica, R. R. (1979). Accuracy of phonotaxis in the green treefrog (Hyla cinerea). Journal of Comparative Physiology A, 133, 247-255.

Ryan, M. J., \& Cummings, M. E. (2005). Animal signals and the overlooked costs of efficacy. Evolution, 59, 1160-1161.

Schöneich, S., \& Hedwig, B. (2010). Hyperacute directional hearing and phonotactic steering in the cricket (Gryllus bimaculatus deGeer). PLoS One, 5, e15141.

Shen, J., Feng, A. S., Xu, Z., Yu, Z., Arch, V. S., Yu, X., et al. (2008). Ultrasonic frogs show hyperacute phonotaxis to female courtship calls. Nature, 453, 914-916.

Ursprung, E., Ringler, M., \& Hödl, W. (2009). Phonotaxis approach pattern in the neotropical frog Allobates femoralis: A spatial and temporal analysis. Behaviour, $146,153-170$.

Wilson, D. R., \& Mennill, D. J. (2011). Duty cycle, not signal structure, explains conspecific and heterospecific responses to the calls of black-capped chickadees (Poecile atricapillus). Behavioral Ecology, 22, 784-790. 